\title{
Six-Minute Walking Test as a Predictor of Clinical Decompensation in Patients with Cirrhosis
}

\author{
Diane Michela Nery Henrique ${ }^{1}$, Carla Malaguti ${ }^{2}$, Tuany Mageste Limonge ${ }^{3}$, Marcela Rodrigues de Siqueira ${ }^{3,4}$, Thiago \\ Martins Fernandes Paticcie ${ }^{2}$, Pedro Augusto Carvalho Mira $^{3}$, Mateus Camaroti Laterza ${ }^{3}$, Carlos Alberto Mourão \\ Júnior $^{5}$, Fábio Heleno de Lima Pace ${ }^{1}$, Júlio Maria Fonseca Chebli ${ }^{1}$
}

1) Department of Medicine,

Division of Gastroenterology, Federal University of Juiz de Fora, Juiz de Fora;

2) Graduate Program on Rehabilitation Sciences, Federal University of Juiz de Fora, Juiz de Fora, Juiz de Fora;

3) Cardiovascular Research Unit and Exercise Physiology, Federal University of Juiz de Fora, Juiz de Fora;

4) Graduate Program in Public Health, Federal University of Juiz de Fora, Juiz de Fora;

5) Department of Physiology, Federal University of Juiz de Fora, Juiz de Fora, Brazil.

Address for correspondence: Julio Maria Fonseca Chebli, MD, PhD.

Rua Maria José Leal, 296- Juiz de Fora, MG, Brazil

CEP 36036-247

chebli@globo.com

Received: 15.10 .2020

Accepted: 23.12.2020

\section{ABSTRACT}

Background \& Aims: The 6-minute walk test (6MWT) is a measure of the overall functional capacity and is associated with the risk of mortality in patients with liver cirrhosis and in those listed for liver transplantation. Nevertheless, physical performance has not yet been established as a predictor of the risk of clinical decompensation in patients with cirrhosis. We aimed to determine the capacity of the 6MWT to predict the clinical decompensation in patients with cirrhosis after 1 year of follow-up.

Methods: This prospective cohort study included patients with compensated cirrhosis of several etiologies. All participants had stable clinical conditions for at least 6 months prior to baseline. At baseline, patients performed the $6 \mathrm{MWT}$ and were followed up for 1 year to detect the decompensation outcomes.

Results: A total of 55 participants completed the evaluation and follow-up. The mean age was $56.3 \pm 10.5$ years, and 65\% were men. Around $65.4 \%$ were classified as Child-Pugh class A. In the receiver operating characteristic analysis, a walking distance $\leq 401.8 \mathrm{~m}$ during the $6 \mathrm{MWT}$ was set as the threshold for predicting clinical decompensation with $64 \%$ sensitivity and $82 \%$ specificity. Kaplan-Meier curve analysis revealed that patients who covered a distance of $<401.8 \mathrm{~m}$ during the test had a decompensation-free outcome rate of $30 \%$ as compared to the rate of $75 \%$ of those who walked $>401.8 \mathrm{~m}(\mathrm{p}<0.001)$.

Conclusions: The $6 \mathrm{MWT}$ was a significant predictor of clinical decompensation in patients with cirrhosis. A cutoff of $401.8 \mathrm{~m}$ was related to an increased risk of clinical decompensation in cirrhotic patients with a stable clinical condition at baseline. The 6MWT should be added to the clinical assessment of the cirrhotic population.

Key words: liver cirrhosis - 6-minute walk test - clinical decompensation - functional capacity - mortality.

Abbreviations: 6MWT: 6-minute walk test; BMI: body mass index; CI: confidence interval; INR: international standardized ratio; NASH: nonalcoholic steatohepatitis.

\section{INTRODUCTION}

Cirrhosis is a chronic disease that involves an anatomical architectural distortion and functional impairment of the liver [1]. The Global Burden of Disease Study data show that 1.3 million people died of liver cirrhosis worldwide in 2015. This value is close to that attributed to diabetes mellitus (1.5 million deaths) and chronic renal failure $(1.2$ million) [2]. Liver cirrhosis has an insidious and long asymptomatic phase (characterized as a compensated stage) until its progressive evolution toward clinical decompensation, which is manifested by ascites, variceal bleeding, encephalopathy, and jaundice. In the decompensation stage, cirrhosis follows an unfavorable and accelerated course toward the need for transplantation or death [1].

Transition to a decompensated cirrhosis stage occurs at a rate of 5-7\% per year [3]. Due to the high burden imposed by clinical decompensation, its prevention is a major target of the current management strategies for liver cirrhosis. In contemporary cirrhosis guidelines, decompensation is used to guide the choice of the initial and subsequent treatments [4]. Therefore, it is necessary to devise tools that can predict the deterioration of the disease. The 6-minute walk test (6MWT) is a simple and widely used measure for evaluating the clinical status of several chronic cardiopulmonary conditions $[5,6]$. This test has recently been used to identify candidates for liver transplantation [7] and to evaluate the risk of mortality in cirrhosis. It has also been associated with the occurrence of the hepatopulmonary syndrome $[8,9]$. 
From this perspective, it is compelling to test the predictive validity of the $6 \mathrm{MWT}$ for identifying patients with cirrhosis who are most likely to present clinical decompensation in the short- and medium term of the disease. Therefore, this study aimed to determine whether the 6MWT could predict clinical decompensation in patients with liver cirrhosis who were followed over a 12-month period.

\section{METHODS}

This prospective, longitudinal, observational study assessed the ability of the 6MWT to predict clinical decompensation of liver cirrhosis in the short- and medium terms of the disease. Clinical decompensation of liver cirrhosis during follow-up was defined as the development of any of the following signs or symptoms: jaundice, ascites, bleeding from esophageal varices, hepatic encephalopathy, or spontaneous bacterial peritonitis. The study was conducted between January 2018 and December 2019 on adult outpatients. It was approved by the institutional Ethics Committee (number 2.458.177). All patients voluntarily signed an informed consent form before being included in the investigation.

The inclusion criteria were as follows: 1) diagnosis of liver cirrhosis established by clinical, laboratory, and imaging data or liver histology, 2) age between 18 and 80 years, 3) cirrhosis classified as compensated based on the absence of or a previous history of variceal bleeding, ascites, jaundice, or encephalopathy in the last 6 months. The exclusion criteria were as follows: 1) history of clinical decompensation of liver cirrhosis in the preceding 6 months, 2) surgery in the last 6 months, 3 ) class II or III obesity (body mass index [BMI] $\geq$ $35 \mathrm{~kg} / \mathrm{m}^{2}$ ), 4) human immunodeficiency virus co-infection, 5) hepatocarcinoma, 6) significant concomitant cardiac diseases including ventricular or atrial arrhythmia as detected on clinical examination or baseline electrocardiogram, 7) neuromuscular disorders, 8) other associated underlying chronic diseases, 9) malignancy (except non-melanoma skin cancer), 10) hemoglobin level below $11 \mathrm{~g} / \mathrm{dL}$ at baseline, 11) participation in a formal exercise training program in the preceding 6 months, 12) pregnancy, and 13) any physical limitations that would make walking impossible.

The first phase included clinical evaluation with history-taking and physical examination to determine the co-morbidities and to certify the physical and cognitive conditions for performing the walk test. During this assessment, anthropometric data, cirrhosis etiology, and the Child-Pugh classification were recorded. A conventional resting electrocardiography was performed. Blood tests were performed on the day of the evaluation, and included serology for the human immunodeficiency virus, international standardized ratio (INR), and the bilirubin, albumin, platelets, aminotransferases and creatinine levels.

Two 6MWTs were performed indoors along a flat, straight 50-m corridor supervised by a trained researcher. Heart rate, pulse oxygen saturation, arterial blood pressure, and perception of symptoms according to the Modified Borg Scale [10] were recorded at rest, immediately after the test, and after the first minute of recovery.

The test speed was not controlled and was determined by the participants themselves. Participants were instructed to walk for 6 minutes to as far as they could, without running. They received standardized instructions before the start of the test and verbal feedback at each minute of the test, in accordance with the American Thoracic Society guidelines [11]. Of the two 6MWTs, the one in which a longer 6-minute walk distance was achieved was considered the better one, and was thus, used for the analysis.

Follow-up information on new clinical decompensation episodes was collected by the research assistant during outpatient visits and/or via a phone call with the participants or their relatives at four distinct time points, namely $3,6,9$, and 12 months after the $6 \mathrm{MWT}$ was conducted. The presence or absence of clinical decompensation, as well as information on the requirement of hospitalization, was registered throughout the 12-month follow-up.

For statistical analyses we used the PASS software, version 11 , to evaluate the sample size required for performing the receiver operating characteristic (ROC) curve analysis. Using a two-sided z-test at a significance level of 0.05 , a sample of 30 patients from the group with decompensation and 20 patients from the group without decompensation achieved $99 \%$ power for detecting a difference of 0.30 between an area under the ROC curve (AUC) of 0.50 under the null hypothesis and an AUC of 0.80 under the alternative hypothesis. The data comprised of continuous responses. The AUC is computed between false positive rates of 0 and 1 . Moreover, a total sample size of 50 (split equally between the two groups), or 25 events, achieves $100 \%$ power to detect a hazard rate of 1.7937 when the proportions surviving in each group are 0.60 and 0.40 , using a two-sided log rank test [12].

The Kolmogorov-Smirnov test was used to test the normality of the data. A descriptive analysis was performed. Continuous variables are presented as mean \pm standard deviations, while categorical variables as frequencies or percentages. The inter- group differences in the presence or absence of any clinical decompensation throughout the 12-month follow-up period were evaluated using independent t-tests. Patients were divided into two subgroups according to whether decompensation occurred or not during the follow-up: patients with clinical decompensation that is that presented during 12 months follow-up with any of the following findings: jaundice, ascites, bleeding from esophageal varices, hepatic encephalopathy, or spontaneous bacterial peritonitis, while the other group comprised of those without any decompensation.

The possible associations between categorical variables and the outcome (i.e., presence or absence of decompensation) were verified using the $\chi^{2}$ test. The sensitivity and specificity of the 6MWT were determined using the ROC curves, AUC curves, and their respective 95\% confidence intervals (CIs). The cutoff $6 \mathrm{MWT}$ value that best predicted the presence or absence of decompensation was determined using the Youden index [13] and effectiveness parameters [14]. To illustrate the differences in the occurrence of clinical decompensation over time, Kaplan-Meier curves were constructed for cumulative survival in consideration of the 6MWT cutoff point found on the ROC curve. To compare the differences between these curves, we used the log-rank test. All tests were two-tailed, and p-values $<0.05$ were considered significant. Data were analyzed using SPSS Statistics, version 26.0 (Armonk, NY). 


\section{RESULTS}

A total of 450 patients with liver cirrhosis underwent screening for the inclusion criteria. Among these, 102 were eligible, and 66 of these patients agreed to participate in the research and were referred for initial evaluation. However, nine (13.6\%) patients were not enrolled, because they: 1) gave up on performing the $6 \mathrm{MWT}(\mathrm{n}=5), 2)$ had difficulty walking due to an osteoarticular cause $(\mathrm{n}=2)$, or 3$)$ presented an underlying cardiac disease $(\mathrm{n}=2$; aortic stenosis and chronic coronary insufficiency). In addition, two (3\%) patients were lost to follow-up. The remaining 55 (83.3\%) participants completed the evaluation and the follow-up of the study. Fig. 1 shows the flowchart of the participants during the study.

The baseline characteristics of the participants are shown in Table I. The mean age was $56.3 \pm 10.5$ years, and $65.5 \%$ were men. No patient was classified as Child-Pugh class C, whereas most patients were classified as class $A(n=36 ; 65 \%)$. The most common etiologies for cirrhosis were alcohol, hepatitis $\mathrm{C}$ virus infection, and nonalcoholic steatohepatitis (NASH). Patients classified as Child-Pugh class A and B were similar in terms of their body mass index (BMI). The mean age of the patients classified as Child-Pugh class A was greater than that of those classified as Child-Pugh class B. As expected, patients classified as Child-Pugh class B had lower levels of albumin, higher levels of bilirubin, and a higher INR than those classified as ChildPugh class A. At baseline, the exercise capacity (as assessed using the 6MWT) did not differ between the patients classified as Child-Pugh classes A and B.

A total of $22(40 \%)$ participants presented with decompensation during the follow-up period. Child-Pugh class B was more predominant in the group that presented with decompensation as compared to the group that did not (54\% vs. $22 \%, p=0.01$, respectively) (Table II). Clinical decompensations observed during the follow-up included the onset of jaundice $(n=6)$, upper gastrointestinal bleeding from esophageal varices $(\mathrm{n}=5)$, ascites $(\mathrm{n}=4)$, and spontaneous bacterial peritonitis $(\mathrm{n}=2)$. During this period, seven (13\%) patients were hospitalized. Their performance on the 6MWT was significantly lower $(328.2 \pm 77.3$ $\mathrm{m})$ than that of those who were not hospitalized (418.8 $\pm 95.6 \mathrm{~m}$;

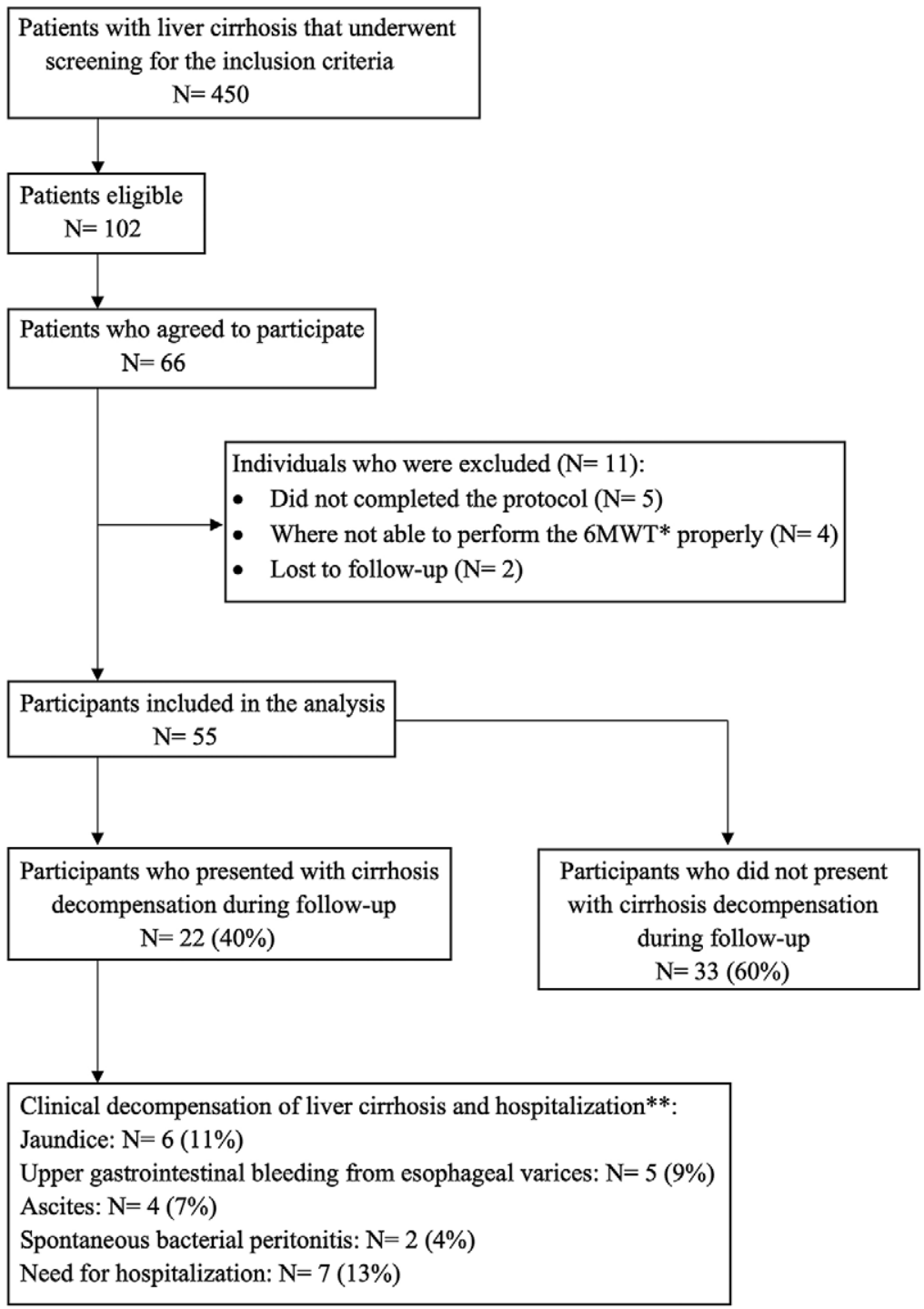

Fig. 1. Flow chart of the participants. ${ }^{*} 6 \mathrm{MWT}$ : 6 -minute walk test; ${ }^{*}$ Some patients experienced more than one of these conditions. 
Table I. Clinical and anthropometric characteristics of the patients.

\begin{tabular}{lcccc}
\hline Variables & All $\mathrm{n}=55$ & $\begin{array}{c}\text { Child-Pugh class A } \\
\mathrm{n}=36(65.4 \%)\end{array}$ & $\begin{array}{c}\text { Child-Pugh } \\
\text { class B }=19(34.6 \%)\end{array}$ & $\mathrm{p}$ \\
\hline Gender (M/F) & $36 / 19$ & $24 / 12$ & $12 / 7$ & 0.51 \\
Age (years) & $56.3 \pm 10.5$ & $58.5 \pm 8.7$ & $52.2 \pm 12.4$ & $0.03^{*}$ \\
Body mass index (kg/m²) & $28.6 \pm 5.5$ & $28.3 \pm 5.6$ & $29.1 \pm 5.5$ & 0.65 \\
Etiology of cirrhosis & & & & 0.09 \\
$\quad$ Alcohol & $20(36.4 \%)$ & $10(27.8 \%)$ & $10(52.6 \%)$ & \\
$\quad$ Virus & $13(23.6 \%)$ & $11(30.5 \%)$ & $2(10.5 \%)$ & \\
$\quad 11(20 \%)$ & $7(19.5 \%)$ & $4(21.2 \%)$ & \\
$\quad$ NASH & $11(20 \%)$ & $8(22.2 \%)$ & $3(15.8 \%)$ & \\
$\quad$ Others & $1.7 \pm 1.4$ & $1.4 \pm 1.2$ & $2.3 \pm 1.6$ & $0.02^{*}$ \\
Total bilirubin (mg/dL) & $3.6 \pm 0.6$ & $3.9 \pm 0.4$ & $3.0 \pm 0.4$ & $<0.01^{*}$ \\
Albumin (g/dL) & $49.9 \pm 30.3$ & $41.6 \pm 21.1$ & $65.8 \pm 38.5$ & $<0.01^{*}$ \\
AST (U/L) & $39.4 \pm 27.0$ & $35.7 \pm 25.3$ & $46.3 \pm 29.5$ & 0.17 \\
ALT (U/L) & $1.3 \pm 0.4$ & $1.2 \pm 0.2$ & $1.5 \pm 0.7$ & $<0.01^{*}$ \\
INR & $93,583 \pm 44,292$ & $95,223 \pm 42,553$ & $90,475 \pm 48,464$ & 0.71 \\
Platelets (/mm3) & $0.9 \pm 0.6$ & $0.8 \pm 0.8$ & $1.2 \pm 1.0$ & $0.02^{*}$ \\
Creatinine (mg/dL) & $434 \pm 105$ & $451 \pm 95$ & $401 \pm 117$ & 0.9 \\
6MWT (m) &
\end{tabular}

Data are presented as mean \pm standard deviation and frequencies (\%). NASH: nonalcoholic steatohepatitis; ALT: alanine aminotransferase; AST: aspartate aminotransferase; INR: international standardized ratio; 6MWT, 6-minute walk test. $\left(^{*}\right)$ indicates significant differences observed between the groups

$\mathrm{p}=0.03$ ). Fig. 2 shows the number and causes of decompensation at each follow-up time point.

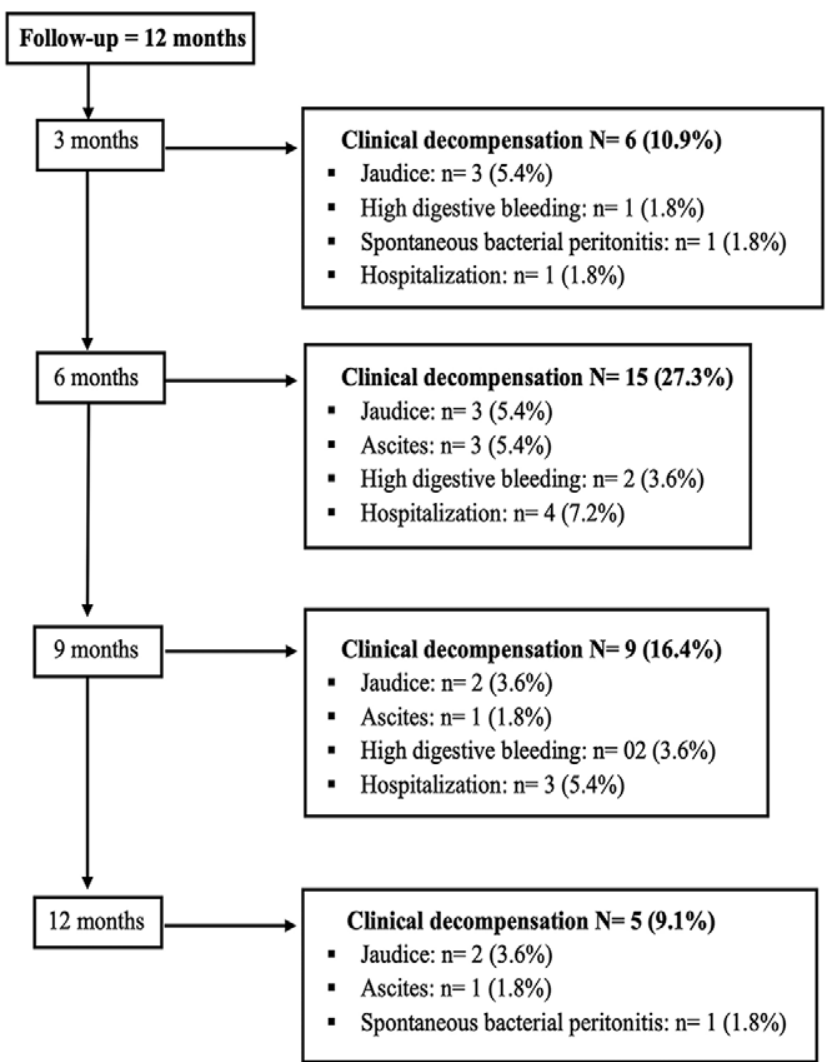

Fig. 2. Flowchart illustrating the numbers and causes of clinical decompensation of liver cirrhosis at each time point during the followup period. ${ }^{*}$ Some patients had more than one episode of clinical decompensation of liver cirrhosis during the follow-up.
Patients who did not present with decompensation of liver cirrhosis walked longer distances during the 6MWT than those who presented with decompensation $(470.9 \pm 76.1 \mathrm{~m}$ and $378.6 \pm 119.1 \mathrm{~m}$, respectively; $\mathrm{p}<0.001$ ) (Table II). The ROC curve indicated a cutoff point of $401.8 \mathrm{~m}$ and an AUC of 0.75 (95\% CI: 0.61-0.88) for discriminating patients who presented with clinical decompensation from those who did not. For this cutoff value, the Youden index [(sensitivity + specificity $)-1]$ was 0.45 and the efficiency (arithmetic mean of the specificity and sensitivity) was 73 (Fig. 3).

Kaplan-Meier survival curve analysis was used to assess the patients with liver cirrhosis in terms of the distance walked (Fig. 4). Patients were dichotomized according to a cutoff of $401.8 \mathrm{~m}$ of distance walked during the 6 MWT $(<401.8$ or $>$ $401.8 \mathrm{~m}$ ). Log-rank test showed that those who walked $<401.8$ $\mathrm{m}$ were more likely to present with clinical decompensation at 12 months of follow-up $(\mathrm{p}<0.01)$ than those who walked $>401.8 \mathrm{~m}$, suggesting that clinical decompensation is linked to lower exercise capacity.

\section{DISCUSSION}

This study showed that the 6MWT is a feasible, safe, and acceptable test for adults with liver cirrhosis. The main findings suggest that patients with compensated cirrhosis who walked $<401.8 \mathrm{~m}$ in the 6MWT may have a higher risk of clinical decompensation of cirrhosis in the short- and medium-term and that preventive strategies should be particularly intensified in such patients.

Clinical decompensation is a landmark in patients with cirrhosis because it represents a new stage characterized by a drastic drop in the survival rate by around 2 years [3]. The clinical features of cirrhosis include progressive periods of 
Table II. Comparison of inter-group differences in the presence or absence of clinical decompensation during the 12-month follow-up.

\begin{tabular}{|c|c|c|c|}
\hline Variables & Without clinical decompensation $(n=33)$ & Clinical decompensation $(n=22)$ & $\mathrm{p}$ value \\
\hline Gender (M/F) & $20 / 13$ & $16 / 6$ & 0.35 \\
\hline BMI $\left(\mathrm{kg} / \mathrm{m}^{2}\right)$ & $29.4 \pm 6.0$ & $27.3 \pm 4.5$ & 0.16 \\
\hline \multicolumn{4}{|l|}{ Etiology of cirrhosis } \\
\hline Alcohol & $8(24.2 \%)$ & $12(54.5 \%)$ & $0.03^{* *}$ \\
\hline Virus & $11(33.3 \%)$ & $2(9.1 \%)$ & - \\
\hline NASH & $5(15.2 \%)$ & $6(27.3 \%)$ & - \\
\hline Others & $9(27.3 \%)$ & $2(9.1 \%)$ & - \\
\hline Child-Pugh class (A/B) & $26 / 7$ & $10 / 12$ & $0.01^{*}$ \\
\hline 6MWT (m) & $470.9 \pm 76.1$ & $378.6 \pm 19.1$ & $<0.001^{*}$ \\
\hline
\end{tabular}

Data are presented as means \pm standard deviations and frequencies (\%). BMI: body mass index; NASH: nonalcoholic steatohepatitis, 6MWT: 6-minute walking test. $\left(^{*}\right)$ indicates significant differences observed between the groups. $\left({ }^{* *}\right) \chi^{2}$ test.

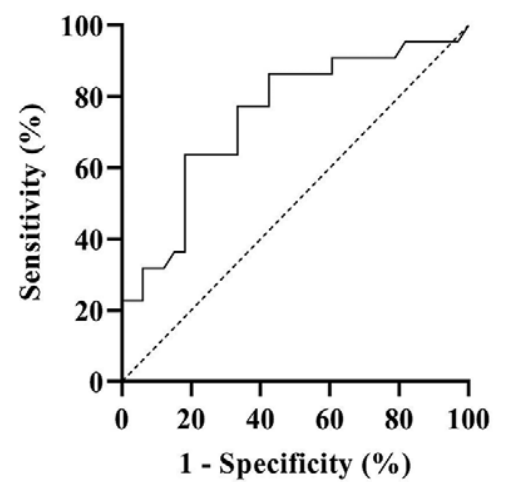

Fig. 3. Receiver operating characteristic (ROC) curve for the cutoff point of the 6-minute walk test to discriminate patients with the presence or absence of decompensation of liver cirrhosis at 1 year of follow-up. The cutoff point was $401.8 \mathrm{~m}$; sensitivity $=64 \%$; specificity $=82 \%$; area under the ROC curve $=0.75$ meters, $95 \%$ confidence interval: 0.61-0.88

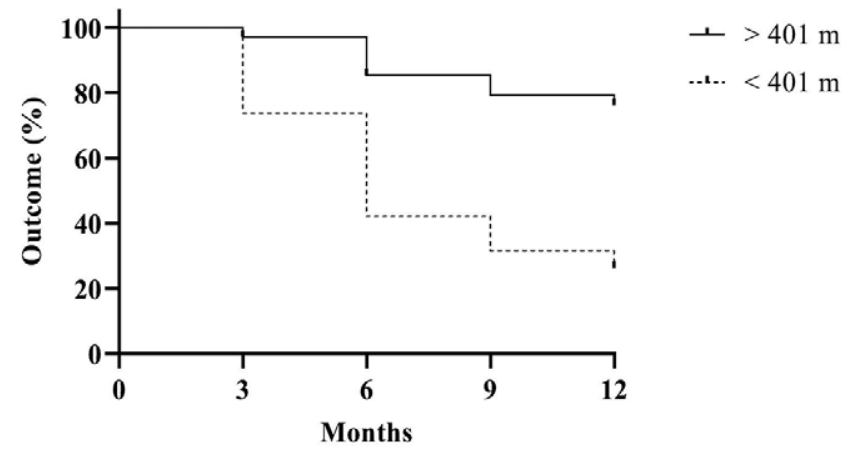

Fig. 4. Participants who walked $<401.8 \mathrm{~m}$ during the 6-minute walk test had a decompensation-free survival rate of $30 \%$ as compared to the $75 \%$ for those who walked $>401.8 \mathrm{~m}$ ( $\mathrm{p}<0.01), 95 \%$ confidence interval: 4.16-7.84.

decompensation and reduced exercise capacity. Although progressive reduction in the liver function and exercise capacity is inevitable, there can be considerable variations in the rate of disease progression among patients. In addition, longitudinal changes can be discrepant between the various measures used to assess clinical status, as periods of transient stability in one marker may be associated with a significant decline in others and vice versa [15]. Faced with a highly variable clinical course, the identification of predictors of clinical decompensation that are accurate and easy to apply in clinical practice is timely and has important clinical implications for the management of patients with cirrhosis [16].

In our sample, the most frequent etiologies of cirrhosis were alcohol-related liver disease, chronic viral hepatitis, and NASH [4]. The patients who presented and did not present with clinical decompensation were similar in terms of the demographic or anthropometric characteristics; however, they differed in the etiology of cirrohosis. Specifically, in the group that did not present with decompensation, there was a higher prevalence of a viral etiology, whereas in the group that presented with decompensation, an alcoholic etiology was more prevalent. This is consistent with the findings of a previous study that showed that the natural evolution of cirrhosis varies with its etiology and that annual rates of decompensation in alcoholic cirrhosis, especially with continuous alcohol use, are higher than those associated with viral etiology [17].

The present study also shows that new decompensation events were significantly more prevalent in the group of patients with a decreased liver functional reserve, as well as in those who covered the shortest distance during the 6MWT (Table II). These results are consistent with those of a remote study involving subjects with more severe cirrhosis who showed poor performance at submaximal exercise [18]. Similarly, Alameri et al. [19] found that these severity characteristics were related to a shorter distance walked in the 6MWT.

Of note, the present study also shows that participants who ambulated less at $6 \mathrm{MWT}$ also had more hospitalizations. This finding is consistent with that also observed in patients with chronic heart failure [20] and chronic obstructive pulmonary disease [21], in which walking distances less than 300 and 350 $\mathrm{m}$, respectively, were independent predictors of hospitalization.

In the present study, we examined the specific contribution of the $6 \mathrm{MWT}$ as a predictor of short- and medium-term clinical decompensation in a representative population of patients with liver cirrhosis. A baseline walking distance in the 6MWT of $<401.8 \mathrm{~m}$ predicted the risk of developing clinical decompensation within a year. To the best of our knowledge, 
this is the first study to describe the use of the 6MWT to predict clinical decompensation in patients with cirrhosis. Interestingly, in agreement with our study, Pereira et al. [9] identified a distance of $<410 \mathrm{~m}$ on the $6 \mathrm{MWT}$ to be a predictor of mortality in a cohort of patients with cirrhosis who were followed up for 3 years. Carey et al. [7] found that the 6MWT was an independent predictor of mortality in candidates for liver transplantation; a distance of $250 \mathrm{~m}$ was the threshold for survival in these patients. It is important to highlight that patients on the waitlist for transplant are usually at an advanced stage of liver disease and present with severe liver dysfunction.

Limitations in performing the 6MWT exercise arise from a dysfunction in the interaction of systems such as the cardiovascular system, respiratory system, and/or peripheral muscle function. Particularly, in cirrhotic individuals, the reduced tolerance to exercise is due to a decreased aerobic capacity and is directly associated with an impaired liver function [22]. It is speculated that the cause of this reduction is multifactorial and involves multiple systems due to port-pulmonary hypertension [8], circulatory circulatory hyperdynamic state [23], physical deconditioning, and sarcopenia [24]. The repercussions of these dysfunctions are clearly manifested in the outcome of 6MWT.

The 6MWT is well-established and is the most commonly used field test to assess the functional capacity in a wide range of severely impaired patients [11]. Furthermore, this test has the advantages of being easy to perform, inexpensive, reliable, and not requiring advanced training or special equipment [11], and can be a major contribution to the daily practice of hepatology. Although the Child-Pugh score is a valid measure to predict the risks in this population, it is subject to variability because the clinical judgment of ascites and encephalopathy can be arbitrary, as can be the cutoff definition in this score. The advantage of detecting the risk of clinical decompensation is having the opportunity to optimize the treatment (including physical rehabilitation) to avoid or delay decompensation. Therefore, we suggest adding the 6MWT to the clinical model to increase the prediction of the informational risk of clinical decompensation in patients with cirrhosis.

Another issue to consider is that due to sarcopenia, physical inactivity, and fatigue, which accompany the progression of liver cirrhosis, a rehabilitation program is necessary for these patients to potentially improve the functional state and change the risk of clinical decompensation.

Our study has some limitations. First, the 1-year followup period was not sufficiently long. Although the advantage of a longitudinal analysis is that changes can be analyzed in individual patients, a longer follow-up period might be more informative. Second, the modest number of patients in the cohort requires other future studies to confirm our important findings.

\section{CONCLUSIONS}

The findings of the present study suggest that the 6MWT predicts the development of clinical decompensation in the short- and medium-term in patients with compensated liver cirrhosis. A clinical risk was identified when the distance covered in the $6 \mathrm{MWT}$ was $<401.8 \mathrm{~m}$, which was predictive for clinical decompensation at 1 year in patients with cirrhosis; the $6 \mathrm{MWT}$ can be used to further refine the prognosis of individual patients and to guide clinical decisions.

Further research and wide implementation of the 6MWT in patients with cirrhosis will help to confirm its validity.

Conflicts of interest: J.M.F.C has served as speaker for Abbott, Abbvie, Janssen, and Takeda. The other authors declared no conflict of interests.

Authors' contribution: M.C.L., F.H.L.P., C.M., J.M.F.C. conceived the study. T.M.L., P.A.C.M., M.C.L., C.A.M.-Jr., F.H.L.P., C.M. designed the methodology. D.M.N.H., M.R.S., T.M.F.P. P.A.C.M performed the investigations. D.M.N.H., T.M.L., M.R.S., T.M.F.P. P.A.C.M: collected data. T.M.L., M.R.S., T.M.F.P., C.A.M.-Jr., analyzed the data. D.M.N.H., T.M.L., J.M.F.C were responsible for the study. C.M., J.M.F.C supervised the study. D.M.N.H., P.A.C.M., M.C.L., C.A.M-Jr., C.M. drafted the manuscript. F.H.L.P., J.M.F.C. edited and critically revised the paper.

Acknowledgement: This study was financed by the Coordenação de Aperfeiçoamento de Pessoal de Nível Superior - Brasil (CAPES) Finance Code 001.

\section{REFERENCES}

1. D'Amico G. The clinical course of cirrhosis. Population based studies and the need of personalized medicine. J Hepatol 2014;60:241-242. doi:10.1016/j.jhep.2013.10.023

2. GBD 2017 Causes of Death Collaborators. Global, regional, and national age-sex-specific mortality for 282 causes of death in 195 countries and territories, 1980-2017:a systematic analysis for the Global Burden of Disease Study 2017. Lancet 2018;392:1736-1788. doi:10.1016/S01406736(18)32203-7

3. D’Amico G, Garcia-Tsao G, Pagliaro L. Natural history and prognostic indicators of survival in cirrhosis: a systematic review of 118 studies. J Hepatol 2006;44:217-231. doi:10.1016/j.jhep.2005.10.013

4. National Institute for Health and Care Excellence. Cirrhosis in Over 16s: assessment and management. Nice guidelines (NG50). 2016. Available at: https://www.nice.org.uk/guidance/ng50

5. Cahalin LP, Mathier MA, Semigran MJ, Dec GW, DiSalvo TG. The sixminute walk test predicts peak oxygen uptake and survival in patients with advanced heart failure. Chest 1996;110:325-332. doi:10.1378/ chest.110.2.325

6. Pinto-Plata VM, Cote C, Cabral H, Taylor J, Celli BR. The 6-min walk distance:change over time and value as a predictor of survival in severe COPD. Eur Respir J 2004;23:28-33. doi:10.1183/09031936.03.00034603

7. Carey EJ, Steidley DE, Aqel BA, et al. Six-minute walk distance predicts mortality in liver transplant candidates. LiverTranspl 2010;16:13731378. doi:10.1002/1t.22167

8. Faustini-Pereira JL, Homercher-Galant L, Garcia E, de MelloBrandão AB, Marroni CA. Exercise capacity of cirrhotic patients with hepatopulmonary syndrome. Ann Hepatol 2015;14:361-368. doi:10.1016/S1665-2681(19)31276-1

9. Faustini Pereira JL, Galant LH, Rossi D, et al. Functional capacity, respiratory muscle strength, and oxygen consumption predict mortality in patients with cirrhosis. Can J Gastroenterol Hepatol 2016;2016:6940374. doi:10.1155/2016/6940374 
10. Borg G. Administration of the Borg Scales. In: Borg G. Borg's perceived exertion and pain scales. 1st Edition. Illinois: Human Kinetics; 1998:4452.

11. ATS Committee on Proficiency Standards for Clinical Pulmonary Function Laboratories. ATS statement:guidelines for the six-minute walk test. Am J Respir Crit Care Med 2002;166:111-117. doi:10.1164/ ajrccm.166.1.at1102

12. Hintze J. PASS 11. NCSS, LLC. Kaysville, Utah, USA. 2011. Available at: http://ncss.com

13. Borges LS. Diagnostic accuracy measures in cardiovascular research. Int J Cardiovasc Sci 2016;29:218-222. doi:10.5935/23594802.20160030

14. Castanho MJ, Barros LC, Vendite LL, Yamakami A. Avaliação de um teste em medicina usando uma curva rocfuzzy. Biomatematica 2004; 14:19-28.

15. Ratib S, Fleming KM, Crooks CJ, Aithal GP, West J. 1- and 5-year survival estimates for people with cirrhosis of the liver in England, 19982009:a large population study. J Hepatol 2014;60:282-289. doi:10.1016/j. jhep.2013.09.027

16. Abraldes JG, Garcia-Tsao G. Simple clinical tools to predict decompensation in patients with compensated cirrhosis:an unmet need. Clin Gastroenterol Hepatol 2019;17:2179-2181. doi:10.1016/j. cgh.2019.04.026

17. Schuppan D, Afdhal NH. Liver cirrhosis. Lancet 2008;371:838-851. doi:10.1016/S0140-6736(08)60383-9
18. Campillo B, Fouet P, Bonnet JC, Atlan G. Submaximal oxygen consumption in liver cirrhosis. Evidence of severe functional aerobic impairment. J Hepatol 1990;10:163-167. doi:10.1016/0168-8278(90)90046-t

19. Alameri HF, Sanai FM, Al Dukhayil M, et al. Six minute walk test to assess functional capacity in chronic liver disease patients. World J Gastroenterol 2007;13:3996-4001. doi:10.3748/wjg.v13.i29.3996

20. Pollentier B, Irons SL, Benedetto CM, et al. Examination of the six minute walk test to determine functional capacity in people with chronic heart failure:a systematic review. Cardiopulm Phys Ther J 2010;21:13-21. doi:10.1097/01823246-201021010-00003

21. Beghé B, Fabbri LM, Garofalo M, et al. Three-year hospitalization and mortality in elderly smokers with chronic obstructive pulmonary disease or chronic heart failure. Respiration 2019;97:223-233. doi: $10.1159 / 000492286$

22. Dharancy S, Lemyze M, Boleslawski E, et al. Impact of impaired aerobic capacity on liver transplant candidates. Transplantation 2008;86:10771083. doi:10.1097/TP.0b013e318187758b

23. Møller S, Bendtsen F, Henriksen JH. Splanchnic and systemic hemodynamic derangement in decompensated cirrhosis. Can J Gastroenterol 2001;15:94-106. doi:10.1155/2001/603012

24. Duarte-Rojo A, Ruiz-Margáin A, Montaño-Loza AJ, Macías-Rodríguez RU, Ferrando A, Kim WR. Exercise and physical activity for patients with end-stage liver disease:improving functional status and sarcopenia while on the transplant waiting list. Liver Transpl 2018;24:122-139. doi:10.1002/lt.24958 